\title{
Research on accumulation and change rule of sucrose in Helianthus tuberosus Linn. under PEG stress
}

\author{
Meng-liang ZHAO ${ }^{1, ~ a ~}{ }^{*}, \mathrm{Li}$-hui WANG ${ }^{2, \mathrm{~b}}$, Xue-mei SUN ${ }^{3, \mathrm{c}}, \mathrm{Yi} \mathrm{LI}^{4, \mathrm{~d}}, \mathrm{Li} \mathrm{LI}{ }^{5, \mathrm{e}}$. \\ ${ }^{1}$ Qinghai Academy of Agricultural and Forestry Sciences,Xining,810016,China; \\ ${ }^{2}$ Qinghai Provincial Key Laboratory of Vegetable genetics and Physiology,Xining,810016,China \\ a8304269@163.com, 'b26079124@qq.com, 'sunxuemei1008@163.com, dy525414@sina.com, \\ eyslili@163.com,
}

Keywords: Helianthus tuberosus L., PEG, Content of sugar, Change rule.

Abstract. Based on the "Qingyu No.1" and "Qingyu No.2 "the two Jerusalem artichoke varieties as experimental materials, the Jerusalem artichoke with different concentration of polyethylene glycol,PEG- 6000 stress $(0,10 \%, 20 \%, 10 \%)$ of drought stress on the content of the different parts of the sugar in the treatment of the different time after change were studied; The results showed that: under the stress of PEG, the sugar content in leaf, Qingyu No.1 and Qingyu No.2 all time increases as the stress increases with increasing time; The content in stem, Qingyu No.1 changes in different with stress time increasing, Qingyu No.2 in PEG concentration was 30\% and stress $24 \mathrm{~h}$ the effect is most obvious; the content in root, Qingyu No.1 and Qingyu No.2 with the increase of stress time its content increased.

\section{Introduction}

Jerusalem artichoke (Helianthus tuberosus L.) is the perennial herbaceous plants that belongs to gene Helianthus of family composite, it native to North America, the edible part of Jerusalem artichoke underground tubers which is rich of inulin. Now the inulin can extend to the development of biomass energy, during the Eleventh Five-Year, China has listed the Jerusalem artichoke as one of the most development value of the biomass energy crops. Because of Jerusalem artichoke with strong resistance, able to adapt to the bad environment, such as drought, cold and salinity, now it has been widely used in ecological management ${ }^{[1]}$, biological energy development ${ }^{[2]}$.

Drought stress can make the corresponding changes in sugar metabolism in plant. Under drought stress, the accumulation of sugar in different parts of Jerusalem artichoke mainly content soluble total sugar, sucrose, glucose and fructose, under drought stress to make sense of the change of sugar in the Jerusalem artichoke body composition and its content it help to explain theoretically that the drought water plant sugar transport in the body and maintain the organization grow sugar metabolic reactions, and regulation of photosynthesis and respiration metabolism and the expression of genes involved in carbon metabolism ${ }^{[3]}$.

The seedlings of Qingyu No.1 and Qingyu No.2 were chosen as materials, using the method of different concentration PEG-6000 simulated drought stress,research on the effects of sugar content changes under the water stress in different parts of Jerusalem artichoke, in order to provide theoretical basis for the regulation of growth under the dry condition of Jerusalem artichoke.

\section{MATERIALS AND METHODS}

\section{Experimental material}

Qingyu No.1 and Qingyu No.2,Jerusalem artichoke varieties were bred autonomously by Qinghai Acad-emy of Agricultural and Forestry Science.

The experimental time and place

Experiments begin from June 2013 to October in horticultural greenhouse of Qinghai Academy of Agricultural and Forestry Science. 


\section{Experiment method}

Using design of two factors experiment, the tubers of Jerusalem artichoke deal with tap water and distilled water rinsed clean and the surface sterilization with $0.05 \%$ of fungicides. Take after the sterilization of terminal bud to pot filled with quartz sand, surface covered quartz sand $1.5 \mathrm{~cm}-2.0 \mathrm{~cm}$. Watering Hoagland nutrient solution every other day, leaching adequately, natural light. After being the third leaf grow, choose the grow even and same size plants as experimental and according to the experimental treatment are numbered,30d after emergence, stress treatments in accordance with the CK（0\% PEG）, treatment 1（10\% PEG）, treatment 2（20\% PEG）, treatment 3 (30\% PEG) ,each processing repeat 3 times, and each time processing are $200 \mathrm{ml}$ dose.

The content of total sugar was determined by glucose oxidasedetection,the content of Glucose, fructose, sucrose and fructan were determined by HPLC.

\section{Data Analysis}

Using DPS software to deal with the data and analy-sis, Using Excel to charting.

\section{RESULTS AND ANALYSIS}

Under different stress of PEG treatment on the impact of the sugar change in Jerusalem artichoke leaf

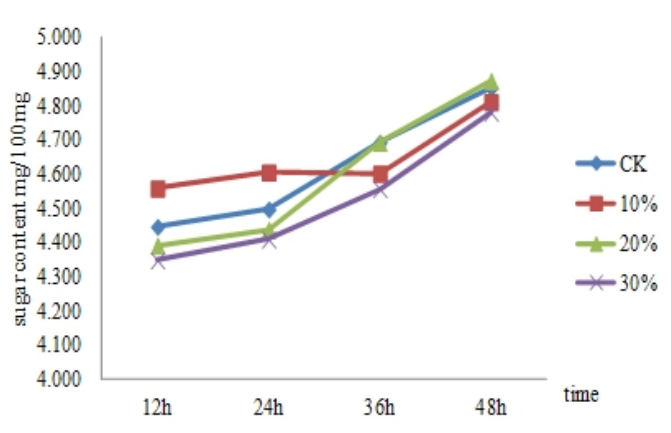

Fig 1

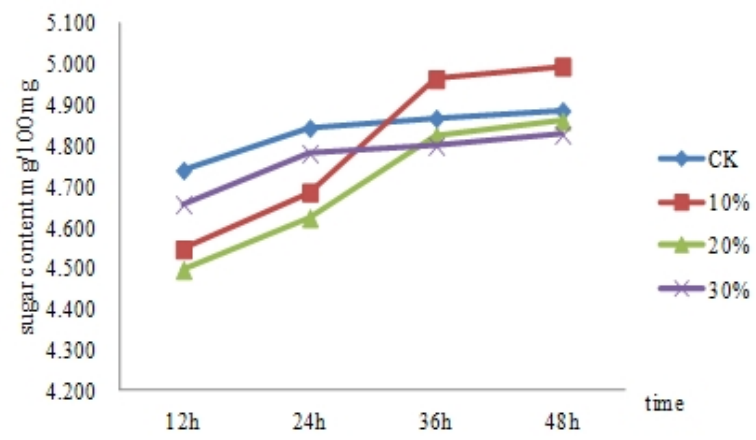

Fig 2

Fig 1 The change trend of sugar content in the leaf of Qingyu No.1 under PEG stress

Fig.2 The change trend of sugar content in the leaf of Qingyu No.2 under PEG stress

As shown in Fig.1,under different concentrations of PEG stress the sugar content in leaf of Qingyu No.1 was increasing as the stress time increasing. In the early stage of the stress, under $10 \%$ PEG concentra-tion stress the sugar content in leaf are higher than CK, sugar content in leaves under $20 \%$ and 30\% concentration PEG stress are lower than CK, stress in the late, sugar content in leaves under different concentrations of PEG stress are all lower than the control, it shows that high concentration and long time of PEG stress might hinder the accumulation of sugar in the leaves.

As shown in Fig.2, under different concentrations of PEG stress the sugar content in leaf of Qingyu No. 2 was increasing as the stress time increasing. In the early stage of the stress, under $10 \%, 20 \%, 30 \%$ PEG concentration stress the sugar content in leaf are higher than CK, and with the increase of stress con-centration, sugar content in the leaves shows that first rise after falling, stress in the late, sugar content in leaves under 10\% concentrations of PEG stress are higher than the control,20\% and 30\% concentra-tions of PEG stress are all lower than the control. 
Under different stress of PEG treatment on the impact of the sugar change in Jerusalem artichoke stem

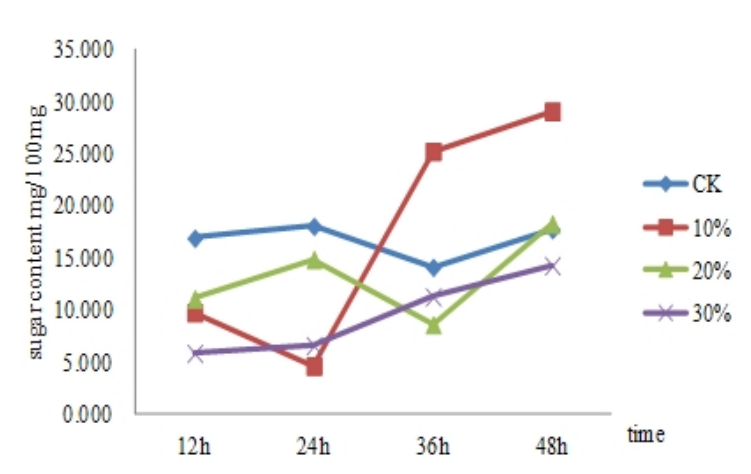

Fig 3

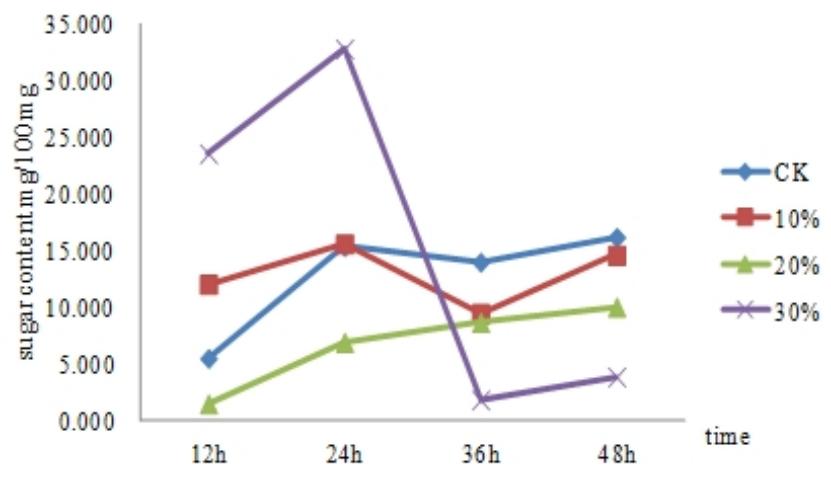

Fig 4

Fig.3 The change trend of sugar content in the stem of Qingyu No.1 under PEG stress

Fig.4 The change trend of sugar content in the stem of Qingyu No.2 under PEG stress As shown in Fig.3, the change trends of sugar content in stem of Qingyu No.1 shows different, under different concentrations of PEG stress as the stress time increase. With the increase of stress time, sugar content in the stem basic remain unchanged under CK and 10\% PEG stress; the sugar content in stem decline as time increasing under 20\% PEG stress, but at the beginning of the stress, the sugar content in the stem are significantly higher than other processing; Under 30\% PEG stress, the sugar content in the stem of Qingyu No.1 is increasing with the stress time and content quickly increase to $11.30 \mathrm{mg} / 100 \mathrm{mg}$ at $48 \mathrm{~h}$.

As shown in Fig.4, at the beginning of stress, differ-ent concentrations of PEG stress can effectively in-crease the sugar content in the stem of Qingyu No.2, the degree of effect is $10 \%<20 \%<30 \%$, and in $30 \%$ PEG concentration and when time for $24 \mathrm{~h}$, the stress effect is most obvious, the sugar content in stem is $2.91 \mathrm{mg} / 100 \mathrm{mg}$,With the increase of stress time, under $10 \%$ and $20 \%$ concentrations of PEG stress and when time for $48 \mathrm{~h}$ the sugar content lower than control,however,30\% concentrations of PEG stress and when time for $36 \mathrm{~h}$ the sugar content lower than control.

Under different stress of PEG treatment on the impact of the sugar change in Jerusalem artichoke root

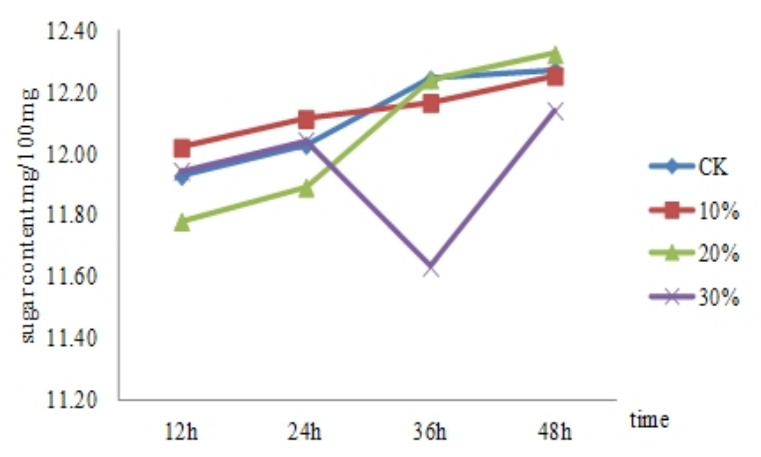

Fig 5

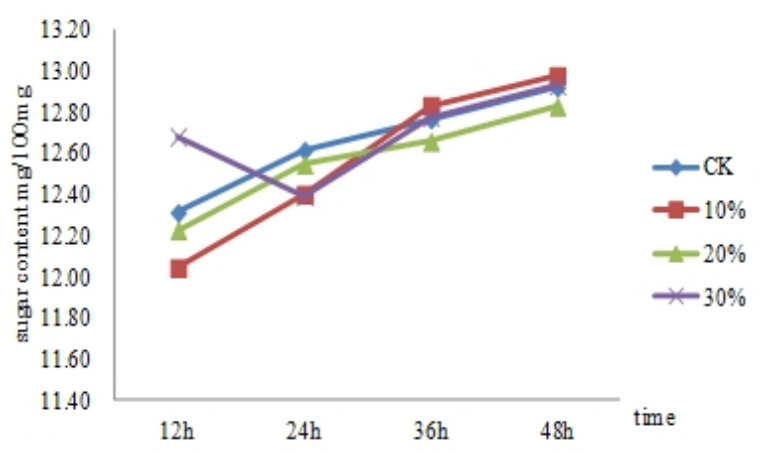

Fig 6

Fig. 5 The change trend of sugar content in the root of Qingyu No.1 under PEG stress

Fig.6 The change trend of sugar content in the root of Qingyu No.2 under PEG stress

As shown in Fig.5, in addition to the 30\% concentration PEG stress, the sugar content Qingyu No.1 are all increasing with the stress time. The diagram also can be seen that at the beginning of PEG stress the different concentrations of PEG stress all can improve the sugar content in root, in the late stress, the sugar content in toot was lower than control under all different PEG concentrations stress.

As shown in Fig.6, under different concentrations of PEG stress the sugar content in root of Qingyu No. 2 were increasing with stress time, but in addition to the concentration of $30 \%$ PEG stress, sugar content in root in the stress period were higher than $\mathrm{CK}$, and stress concentration effects on the sugar content in root was $10 \%<20 \%$.In the late stress, $20 \%$ concen-tration of PEG stress basically have no impact on sugar content in root. 


\section{Summary and discussion}

The soluble sugar abundant accumulation in plants under drought stress, one hand can reduce cell os-motic potential in order to maintain cell turgor pres-sure, to prevent a large number of passive dehydra-tion in the cell ${ }^{[4]}$ on the other hand, excessive accumulation of soluble sugar often feedback inhibi-tion on photosynthesis ${ }^{[5]}$, Such as moderate drought stress can control wheat stalks carbohydrate content increased, Stimulate its to grain transshipment, so as to improve the ability of wheat against drought stress ${ }^{[6-7]}$,Yan $\mathrm{G}$. et $\mathrm{al}^{[8]}$, done the effects of exogenous spermidine on metabolism of nonstructural carbonhydrate and involved activity of enzymes of tomato seedlings under drought stress,results showed that exogenous spermine influence carbohydrate content, participated in the tomato seedlings under drought stress carbohydrate metabolism; Yang G.T. et $\mathrm{al}^{[9]}$ research on the effects of drought Stress on the carbohydrates distribution and effective constituent contents of Tamarisk Chinese and Cistanche Tubulosa, results showed that soil drought caused the transport proportion of Cistanche tubulosa decreased which transported by the assimilation product of host Tamarix chinesis and the biomass of Cistanche tubulosa declined.

During the process of the Jerusalem artichoke growth and development, a large number of water soluble sugar synthesis and accumulation, and stored in the tuber, main ingredients for sucrose, fructose, glucose and Degree of Polymerization (DP) different fructan, the highest content is fructan, which content can reach more than $80 \%$ of the dry weight of the tuber ${ }^{[10]}$. Soluble sugar as the important osmotic ad-justment matter of plant under drought stress, will increase significantly under osmotic stress, in order to increase in plant osmotic potential, enhance the drought resistance ability of plants ${ }^{[1]}$.Early,part of the author in this paper, had studied on sugar meta-bolism response on drought stress in seedling of Je-rusalem Artichoke ${ }^{[12]}$, the results showed that in a certain extent, drought stress can promote the in-crease of the content of Jerusalem artichoke soluble total sugar and the improve of soluble total sugar was most caused by fructosan.Drought stress by PEG stress experiments showed that:a certain degree of drought stress plays an promoting role in promo-tion of Jerusalem artichoke sugar content, but the content of sucrose, glucose, fructose and sugars in Jerusalem artichoke plants is low, and the period of change happened under drought stress, the intensity and duration of the multiple effects, it is unclear whether several sugars common mechanism of con-version and distribution, the team will continue to carry out to drought stress, which is mainly com-posed of fructan in-depth study of Jerusalem arti-choke sugar response mechanism.

\section{Acknowledgements}

The work was financially supported by the project of Qinghai Academy of Agricultural and Forestry Sciences (2014-NKY-209).

\section{Literature References}

[1] Kai Gao,Tiexia Zhu,Yongliang,Zhang,et al.Effect of Water and Nitrogen on Yield and Biomass Allocation of Helianthus tuberosus. Chinese Journal of Grassland,35(1):49-54 (2013)In Chinese

[2] Zuxi Liu,Guanghui Xie.An overview of researches on Jerusalem artichoke as a biofuel crop .Journal of China Agricultural University,17(6):122-132.( 2012) In Chinese

[3] K. E Koch.Carbohydrate-modulated gene expression in plants.Ann.Rev.Plant Physiol.Plant Mol.Bio. 47:509-540(1996)

[4] T. L.Sanita, R. Gabbrielli.Response to cadmium in higher plants.Environ Exp Bot41:105-130(1999)

[5] C. H.Foyer, M.Lelandais, K. J. Kunert.Photooxidative stress in plants.Physiol Plant,92:693-717(1994) 
[6] B.Ehdaie, GA. Alloush, MA. Madore.Genotypic variation for stem reserves and mobilization in wheat. I.Postanthesis changes in internode dry matter.Crop Science,46:735-746(2006)

[7] Z. Plaut, BJ.Butow, CS.Blumenthal.Transport of dry matter into developing wheat kernels and its contribution to grain yield under post-anthesis water deficit and elevated temperature.Field Crops Research,86:185-198(2004)

[8] Gang Yan,Chunmei Zhang,Zhirong Zou.Effects of exogenous spermidine on metabolism of nonstructural carbonhydrate and involved activity of enzymes of tomato seedlings under drought stress.Agricultural Research in the Arid Areas,30(1):143-148(2012) In Chinese

[9] Guotao Yang,Yuhai Guo,You Du,et al.The Effects of Drought Stress on the Carbohydrates Distribution and Effective Constituent Contents of Tamarisk Chinese and Cistanche Tubulosa. Journal of Anhui Agri Sci,38(26):14246-14247,14249(2010) In Chinese

[10] Xuemei Sun,Lihui Wang,Qiwen Zhong.Study on Change of the Carbohydrates Content of Jerusalem artichoke During Storage.Northern Horticulture,(11):131-134(2012) In Chinese

[11] Yaofu Wang,Shixu Song,Pei Wang,et al.Effect of Osmotic Stress on Physiological Indices of Drought Resistance of Flue-cured Tobacco Leaves under Different Potassium Supplying.Chinese Agricultural Science Bulletin,2(5):216-219(2006) In Chinese

[12] Qiwen Zhong,Lihui Wang,Xuemei Sun,Yi Li,Li Li.Study on Sugar Metabolism Response on Drought Stress in Seedling of Jerusalem Artichoke.Southwest China Journal of Agricultural Sciences, 25(4):1238-1241(2012) In Chinese 